\begin{tabular}{|c|c|c|c|c|c|}
\hline MUNIBE Antropologia-Arkeologia & $n^{\circ} 67$ & $285-293$ & DONOSTIA & 2016 & ISSN 1132-2217 • eISSN 2172-4555 \\
\hline
\end{tabular}

\title{
La gestión de las materias primas silíceas en el Pirineo Occidental: la ocupación Magdaleniense de Berroberria (Urdax, Navarra)
}

\author{
Lehengai silizeoen kudeaketa Mendebaldeko Pirinioetan: \\ Berroberria (Urdax, Nafarroa), Magdalen aldiko egonlekua \\ Siliceous raw material management in Western Pyrenees: \\ Magdalenian occupation of Berroberria (Navarre, Spain)
}

\begin{abstract}
PALABRAS CLAVES: sílex, industria lítica, Paleolítico Superior, petrografía, recursos minerales. GAKO-HITZAK: suharria, industria litikoa, Goi Paleolitoa, petrografia, baliabide mineralak. KEY WORDS: Flint, lithic industry, Upper Palaeolithic, petrography, mineral resources.
\end{abstract}

\section{Irantzu ELORRIETA BAIGORRI ${ }^{(1)}$ y Antonio TARRIÑO VINAGRE ${ }^{(2)}$}

\section{RESUMEN}

La cueva de Berroberria es uno de los yacimientos más representativos del Magdaleniense en el Pirineo Occidental. Se presentan en este caso los resultados del estudio de procedencia de las materias primas líticas empleadas por los grupos del Magdaleniense Medio (alrededor de 14500 cal. BC). La metodología propuesta incluye análisis petrológicos y mineralógicos, mediante los que se identifican los principales afloramientos de sílex de los que se abastecieron. Se ha constatado así la explotación mayoritaria de los recursos silíceos más cercanos, como es el sílex de Flysch, junto con la de recursos regionales, como los sílex de Tercis o Chalosse, y siendo minoritarios pero de gran interés los aportes de materias primas extra-regionales de posible procedencia noraquitana. La gestión de cada una de estas rocas, al igual que su representación, es diferente; pero concuerda con la dinámica observada para esta cronología.

\section{LABURPENA}

Berroberriako kobazuloa Mendebaldeko Pirinioetan Magdalen aldiko aztarnategirik erakusgarrienetariko bat da. Kasu honetan Erdi Magdalen aldiko taldeek (cal BC 14500 inguruan) erabilitako harrizko lehengaien jatorrizko ikerketak aurkeztuko dira. Proposamen metodologikoak azterketa petrologiko eta mineralogikoak sartzen ditu, horien bidez hornitzen zituzten suharrizko iturri printzipalak ezagutuko dira. Honela, hurbil dauden baliabide silizeoen ustiapen gehienena, Flysch-eko suharriena, eskualdeko baliabideekin batera, Tercis edo Chalosse suharriak, egiaztatu da. Eskualdetik kanpoko lehengaiak, Ipar-Akitaniako jatorriak, gutxienenak dira baina interes handikoak. Harrizko lehengai bakoitzaren kudeaketa ez da berdina, bere irudikapenarekin gertatzen den bezala, baina data hauetarako dinamikarekin bat dator.

\section{ABSTRACT}

INTRODUCTION

The Western Pyrenees is an area remarkable for its well-known flint outcrops that were exploited as siliceous raw material sources by the prehistoric societies that occupied the territory. Berroberria cave (Urdax, Navarre) is located in this part of the Pyrenees and it has a large Magdalenian stratigraphy. In this work the results of the siliceous raw materials provenance of the collection of level $\mathrm{G}$ are presented. The selected level dates from the Middle Magdalenian (around 14500 cal BC).

Materials and Methods

Relevant data have been obtained from the archaeological and petrologic analysis of the lithic industry. The employed methodology is based on the identification of the textural macroscopic characteristics determining each flint by stereoscopic microscope and on the observation of thin sections from the siliceous samples under the petrographic microscope. Once they are described, they are compared with the flint samples from the near outcrops in order to confirm its origin. Similarly, the technologic and typological characteristics of the lithic industry are analyzed and later matched up to the information of the siliceous material provenance.

RESULTS

The results are revealing to discern how the resource management by the hunter-gatherer societies of Berroberria was. Firstly, the acquisition points of the siliceous sources have been pointed and defined from the characterization of the elements that integrate the industry. The main flints detected are the coastal Flysch type with two varieties: Bidache and Gaintxurizketa, Tercis, Chalosse and Salies de Béarn located

\footnotetext{
(1) Universidad del País Vasco/Euskal Herriko Unibertsitatea. Facultad de Letras. Departamento de Geografía, Prehistoria y Arqueología. C/ Tomás y Valiente s/n, Laboratorio 1.18A. C.P.: 01006 Vitoria-Gasteiz. Correo electrónico: i_elobai@hotmail.com

(2) Centro Nacional de Investigación sobre la Evolución Humana (CENIEH)
} 
in the north (south Aquitanian Basin) and Urbasa and Treviño from the Basque-Cantabrian Basin at the south. The generated map shows the mobility along a territory that reaches a distance of around 160 kilometres from the site to the most distant outcrop. This area could be enlarged if the provenance of north aquitanian flints is confirmed.

Secondly, it has been evaluated how the diverse flint varieties detected in the site are managed. The concept of "chaîne opératoire" is taken into account in order to recognize the acquisition, configuration and discard stages of the lithic industries. The Flysch flint is the most used and the one that best shows the technological process, therefore the flint that offers more information.

Conclusions

The raw material provenance studies include information about the availability and supplying potential of the territory, drawing the management and mobility map of the societies that inhabited the environment. To sum up, local flints are the most exploited, followed by regional ones and extra-regional materials are slightly represented. The data obtained in this work need to be contrasted with other data of sites of the region. In this way a model of flint exploitation will be obtained for the Western Pyrenees or different patterns limited to smaller areas.

\section{1.- INTRODUCCIÓN}

El Pirineo Occidental destaca por su elevado número de yacimientos prehistóricos conocidos-incluyendo un amplio territorio que se extiende por el sur de la región francesa de Aquitania, departamento de Pyrénées-Atlantiques, y por las provincias del País Vasco y Navarra-. A lo largo de las últimas décadas se han venido realizando diversos estudios, entre los que destacaremos aquellos dedicados a la gestión de los recursos líticos, suscitando interés en la búsqueda de las principales fuentes de abastecimiento (TARRIÑO, 2001a y 2001b; TARRIÑO y NORMAND, 2002; CAVA y BARANDIARÁN, 2008; TARRIÑO y ELORRIETA, 2012; BARANDIARÁN et al. 2013; ARRIZABALAGA et al., 2014; ELORRIETA, 2014; TARRIÑO et al., 2015).

Los resultados han sido beneficiosos pues han suministrado información relevante sobre afloramientos silíceos explotados, localizándolos e identificando los sílex entre las industrias líticas de diferentes yacimientos arqueológicos. Continuando con esta línea de investigación, se presenta el estudio de la procedencia de las materias primas empleadas en la industria lítica del nivel $G$ de Berroberria.

\section{2.- EL YACIMIENTO DE BERROBERRIA (URDAX, NAVARRA)}

El yacimiento de Berroberria se localiza en la cueva del mismo nombre que se encuentra en el municipio de Urdax (Navarra), en la cuenca alta del río Nivelle que desemboca en la localidad francesa de San Juan de Luz en aguas del Atlántico. La cueva está situada en el fondo de una ladera y, a pesar de ser una zona pirenaica, presenta una altitud de 100 m.s.n.m. lo que evidencia su relativa cercanía al mar. Berroberria forma parte del macizo calizo de Celayeta/Berroberria junto con otras cavidades entre la que destacaríamos la de Alkerdi donde también se encontraron evidencias de ocupaciones prehistóricas, además de grabados rupestres paleolíticos.

Las excavaciones en las que se obtuvo el material aquí estudiado se iniciaron en los años 70 de la mano de I. Barandiarán y A. Cava (BARANDIARÁN, 1979 y 1993). La existencia del yacimiento ya era conocida con anterioridad e incluso se habían llevado a cabo intervenciones arqueológicas: en 1939 dirigidas por Marqués de Loriana, en los años 40 por Rivera Manescau, así como bajo el mando de Maluquer de Motes en la década de los 60. El material arqueológico recuperado fue abundante y se obtuvo numerosa información especialmente para cronologías del Paleolítico Superior en el entorno. La estratigrafía de la cueva abarca un amplio intervalo desde el Magdaleniense Antiguo hasta la Edad del Bronce, incluyendo ocupaciones del Magdaleniense Terminal o Aziliense y también Epipaleolíticas (BARANDIARÁN, 1990).

El nivel seleccionado para el estudio de procedencia es el que lleva la designación $G$ y que corresponde con el Magdaleniense Medio (Figura 1). El estrato presentaba un espesor de entre 14 y $22 \mathrm{~cm}$ de tierra marrón, de la que se extrajeron restos de fauna identificándose cérvido, cáprido, bóvido y algún carnívoro. El análisis por datación radiocarbónica que se obtuvo dio

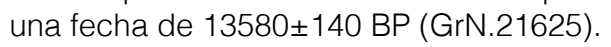

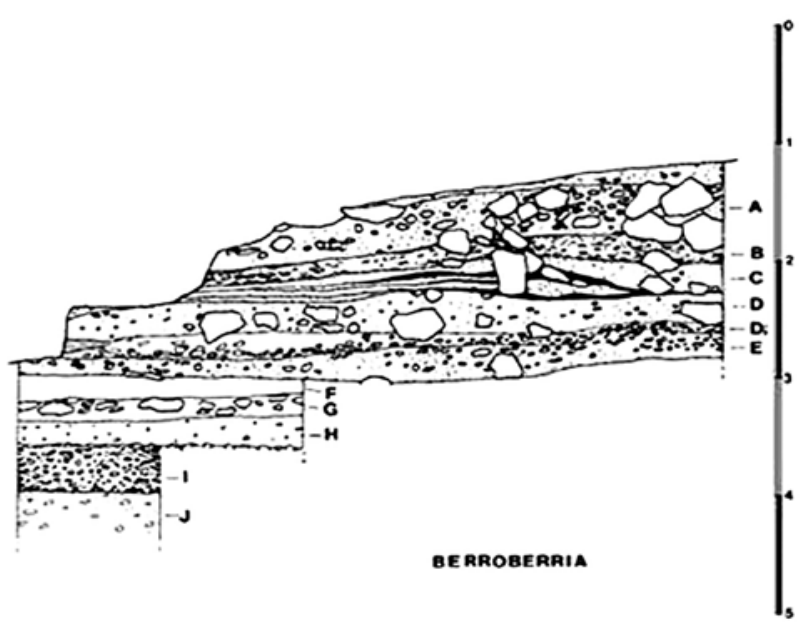

Fig. 1. Estratigrafía de la cueva de Berroberria (según Barandiarán, 1990)/ Stratigraphical section of the excavation in Berroberria (Barandiarán, 1990), levels from Magdalenian to Bronze Age.

\section{3.- METODOLOGÍA Y MATERIAL DE ESTUDIO}

El método empleado en este trabajo es el que venimos empleando (TARRIÑO et al., 2015). Esta propuesta se basa en los estudios propiamente petrológicos y 
mineralógicos, desarrollados por dichas ramas de la Geología, así como la inclusión de análisis geoquímicos que aporten mayor número de información a la caracterización de las rocas silíceas. Sin olvidar el estudio de la industria lítica desde el punto de vista de la Tipología Analítica (LAPLACE, 1973; FERNÁNDEZ-ERASO y GARCÍA-ROJAS, 2013).

\subsection{Análisis llevados a cabo}

El primer acercamiento para la identificación de la materia prima ha sido mediante el empleo de la lupa binocular, con hasta 45 aumentos. Mediante este método se ha caracterizado cada tipo de sílex y se han agrupado, guardando la información en una base de datos.

Por otro lado, se han seleccionado para la realización de láminas delgadas 9 muestras de la litoteca que han servido para realizar el estudio petrográfico en profundidad de los diferentes tipos de sílex.

La información obtenida a través de la petrología ofrece por un lado, datos sobre la difusión, sabiendo la localización de los principales puntos de abastecimiento que facilitan la generación de mapas. Y por otro lado, sobre la gestión a través de la que se llega observando tres aspectos claves de la industria lítica: la tipología, la tecnología y la tipometría. Con todo ello pretendemos reconstruir los comportamientos socio-económicos de las sociedades, en este caso sociedades de cazadores-recolectores del Magdaleniense.

\subsection{Material para el análisis}

El material estudiado del nivel G asciende a 1400 evidencias, correspondientes a las últimas campañas de excavación, de las cuales una décima parte presentaba retoque. Una primera clasificación por materias primas reveló que el $98,7 \%$ era sílex, mientras que el porcentaje restante correspondía a cristal de roca $(1,2 \%)$ y a cuarcita, apenas representada.

\section{4.- RESULTADOS}

Los resultados de la determinación de los sílex se han logrado esencialmente a través de la identificación de los fósiles característicos de cada variedad, puesto que es el distintivo más fiable para ello, unidos a las texturas y composición de las rocas (TARRIÑO, 2001a, TARRIÑO et al., 2015).

\subsection{Principales tipos de sílex}

La determinación de procedencia se ha podido llevar a cabo con seguridad en un elevado porcentaje, en torno al $85 \%$, quedando dentro del grupo de los indeterminados el 15\% restante, es decir, aquellos cuya identificación es dificultosa debido principalmente a su conservación. Las alteraciones que dañan los sílex pueden ser postdeposicionales, provocadas con el paso del tiempo: patinación principalmente, o por acciones antrópicas como el fuego. Estos agentes hacen que los sílex pierdan sus características o cambien de color dificultando así su determinación. A continuación pasaremos a describir los principales tipos silíceos identificados en la colección de Berroberria.

\subsubsection{Sillex de Flysch}

Dentro del sílex denominado de Flysch, cuyos afloramientos con cronología Campaniense del Cretácico superior se encuentran en esta formación de Flysch pirenaico, se han podido identificar dos variedades mayoritarias: la de Bidache y la de Gaintxurizketa. La primera se trata de un sílex bioclástico, fino y con alto contenido en espículas de esponja, así como materia orgánica. Al patinarse lo hace en tonos grisáceos. Es el sílex más empleado en el nivel $\mathrm{G}$ de Berroberria, con un $62 \%$, y también aparece recurrentemente en yacimientos de la Cornisa Cantábrica y Pirineo Occidental, por lo que se considera un sílex trazador. (Figura 2.1A y 1B)

La segunda variedad, la de Gaintxurizketa se caracteriza por una textura bioclástica con impurezas carbonatadas en bioturbaciones y con microcristales de dolomita idiomorfa. (Figura 2.2.A y 2.B) Su representación es de en torno al 8\% en el nivel $\mathrm{G}$.

\subsubsection{Sílex de Tercis}

El sílex de Tercis es un tipo difícil de identificar puesto que al patinarse es similar al sílex de Bidache en su aspecto exterior. Tiene una textura bioclástica con abundantes foraminíferos planctónicos (globigerinidos). (Figura 2.4A y 4B). Sus afloramientos de edad Santoniense-Campaniense (Cretácico superior) aparecen al sur de Las Landas (cercanías de Dax, Francia). Entre el material del nivel $G$ se ha confirmado su presencia en un $8 \%$.

\subsubsection{Sílex de Chalosse}

Este tipo de Chalosse es otro de los denominados sílex trazadores. Es muy conocido y fue muy empleado durante la Prehistoria en el área de Las Landas y también se extendió por la cornisa Cantábrica y Pirineos (CHALARD et al., 2010). Posee una textura bioclástica con briozoos y Lepidorbitoides, fósiles que lo caracterizan. (Figura 2.3A y 3B). Sus principales afloramientos de edad Maastrichtiense (Cretácico superior) se sitúan en el anticlinal de Audignon-Montaut (Departamento de Landas, Francia). En este caso aparece escasamente representado entre la industria lítica, con un $4 \%$.

\subsubsection{Sílex de Salies de Béarn}

El sílex denominado Salies de Béarn tiene una textura bioclástica con impurezas carbonatadas en biotur- 
baciones y con globigerínidos como fósil característico. (Figura 2.5A y 5B) Afloran en terrenos del Senoniense (Cretácico superior) de la región de Béarn (Pirineos Atlánticos, Francia). Es una materia prima que no aparece muy representada en los yacimientos y en Berroberria su empleo no es excepcional pues su uso no alcanza el $0,5 \%$.

\subsubsection{Sílex de Urbasa y de Treviño}

Las variedades más propias de la Cuenca Vasco-Cantábrica son las conocidas como sílex de Urbasa y sílex de Treviño, muy empleadas en yacimientos del País Vasco durante la prehistoria. El primero aflora en terrenos del Terciario marino (Thanetiense, Paleoceno) de la Sierra de Urbasa (Navarra) que le da su nombre. Es un sílex bioclástico con Nummulites heberti y espinas de equinodermo (Figura 2.6A y 6B). Y el de Treviño, cuyos afloramientos del Terciario continental (Aquitaniense, Mioceno) se localizan en el sinclinal de Miranda-Treviño (provincias de Burgos y Álava), se trata de un sílex de origen lacustre-palustre con gasterópodos y ostrácodos fundamentalmente (Figura 2.7A y 7B). Ambos tipos silíceos se han identificado en similar porcentaje en el nivel $\mathrm{G}$ de Berroberria, en torno al 1\% respectivamente.

\subsection{Los sílex noraquitanos}

Como novedad para un yacimiento en territorio español se han identificado dos posibles variedades del norte de la Cuenca de Aquitania. Una de ellas (de la que se ha detectado un solo resto correspondiente con un fragmento con retoque) (Figura 2.9A) presenta una textura equivalente a las que presentan los sílex conocidos como «Grain de mil» puesto que posee características propias (CAUX, 2011): textura bioclástica con briozoos, Rotálidos identificados, conteniendo entre un $40 \%$ y un $60 \%$ de granos.

El sílex de «Grain de mil» debe su nombre a R. Simonnet (1982) quien lo llamó así debido a la singular pátina que presenta que se asemeja a granos de mijo. Las referencias al origen geológico de este sílex (BORDES, 2002; JAUBERT et al., 2008) confirman que se trata de un sílex del Santoniense inferior que aflora en alteritas en la región de Ozillac (Charente Marítimo). Entre sus características destaca por tener una textura wackestone a packestone (clasificación Dunham, 1962), de grano grueso (menor de un milímetro) con numerosos bioclastos; también incluye fragmentos de Briozoos y de foraminíferos bentónicos como Nummofallotia, Textuláridos y Rotálidos y en menor medida fragmentos de algas tipo Lithothamniées y restos de Crinoideos. La zona interna tiene un aspecto sacaroideo con menor cantidad de fósiles debido a la recristalización.

Algunos especialistas han considerado que el «Grain de mil» se puede tratar de una pátina que es similar para varios tipos silíceos diferentes (CAUX, 2011) y quizá no tanto para un único sílex. Por ello se debería hablar con cautela sobre la aparición de este tipo en el Pirineo ya que podría ser una variedad mucho más próxima con una patinación similar. Lo que demuestra la necesidad de unas analíticas completas en las que se identifiquen fósiles u otras características más definitorias de este sílex santoniense.

La segunda posible variedad novedosa es la de Grand-Pressigny (Figura 2.8A). El sílex de Grand-Pressigny tiene un origen más lejano que el anterior, pues sus afloramientos se sitúan al sur de la Cuenca de Paris, en el centro de Francia, en la región de Grand-Pressigny (MILLET et al., 1986). Son sílex de edad Turoniense (Cretácico superior) (PRIMAULT, 2003). La variedad denominada "clásica" es de colores marronáceos y textura wackestone (clasificación Dunham, 1962). En algunos casos aparecen zonaciones en distinto tono. El córtex es generalmente fino, de menos de $1 \mathrm{~mm}$ de espesor y demuestra el contacto con la roca encajante calcárea. Es un sílex de formación marina y bioclástico, por ello los fósiles detectados normalmente son abundantes y variados: foraminíferos, briozoos, equinodermos y moluscos. En la colección estudiada se ha identificado una textura similar a la que presentan estos sílex, aunque no se han encontrado ninguno de los fósiles mencionados. Hasta que no se realicen analíticas más precisas no podemos afirmar con toda seguridad que estos sílex sean de tipo "Grand-Pressigny".

Las evidencias sobre este tipo son de tamaño pequeño o micro, rondando los $10 \mathrm{~mm}$, o incluso de no más de $5 \mathrm{~mm}$. Ello hace que los métodos petrográficos no puedan aportan mayor información debido a la pequeña superficie observable. Son un total de 11 piezas entre las que destaca una con retoque y que corresponde con una truncadura y 4 laminitas. Estos restos hacen pensar en la posibilidad de que se traten de residuos de un proceso de reacondicionamiento desarrollado en el lugar; es decir, posiblemente llegó a la cueva algún soporte de mayor tamaño que fue reconfigurado y que no se ha hallado pero del cual existen evidencias. No sería rara la aparición de elementos exóticos procedentes de largas distancias como ya se ha indicado por varios autores para cronologías del Paleolítico Superior, advirtiéndose una mayor aportación de materiales líticos alóctonos probablemente suscitada por un amplio conocimiento del territorio (GENESTE, 1991; FÉBLOT-AUGUSTINS, 1997).

\section{5.- LA GESTIÓN DE LA MATERIA PRIMA, EX- PLOTACIÓN Y MOVILIDAD}

Tras la descripción de los resultados pueden analizarse en dos niveles, por un lado cómo fue la gestión de cada tipo de sílex y por otro cuál fue el territorio de explotación y por tanto los posibles patrones de movilidad o conocimiento de la zona.

En primer lugar, los sílex y sus afloramientos pueden ser clasificados en relación con la distancia entre ellos 

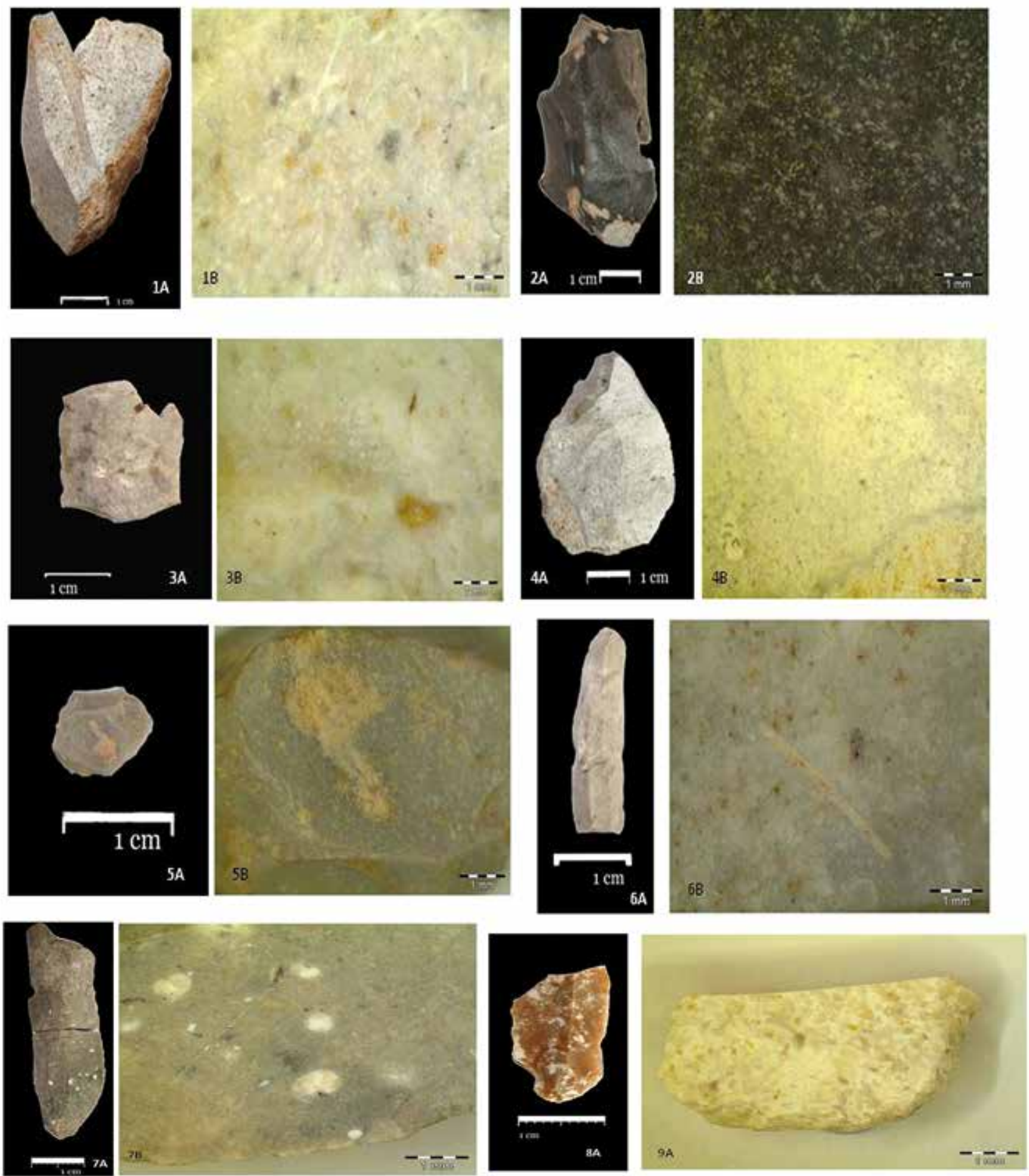

Fig. 2. Fotografías generales y en detalle de los sílex identificados en Berroberria: 1.A. Sílex Flysch de Bidache; B. Detalle de textura bioclástica con espículas de esponja; 2.A. Lasca de sílex de Gaintxurizketa; B. Detalle de la textura bioclástica. 3.A. Fragmento de sílex de Chalosse; B. Detalle de textura bioclástica con briozoos. 4.A. Lasca sílex de Tercis; B. Detalle de textura bioclástica con globigerínido. 5. Fragmento de sílex de Salies de Béarn; B. Detalle de textura bioclástica con bioturbaciones. 6.A. Fragmento de lámina de sílex de Urbasa; B. Detalle de textura bioclástica con una sección de Nummulites heberti. 7.A. Lámina de sílex de Treviño; B. Detalle de textura bioclástica con ostrácodos. 8.A. Aspecto macroscópico de fragmento de sílex posiblemente de tipo Grand-Pressigny. 9.A. Aspecto macroscópico de fragmento de sílex posible "Grain de Mil» / General images and in detail of the flints identified in Berroberria: 1.A. Flysch of Bidache flint; B. Detail of bioclastic texture with sponge spicules; 2.A. Flake of Flysch of Gaintxurizketa flint; B. Detail of bioclastic texture. 3.A. Chalosse flint fragment. B. Detail of bioclastic texture with briozoo. 4.A. Flint flake of Tercis; B. Detail of bioclastic texture with globigerinida. 5.A. Flint fragment of Salies de Béarn; B. Detail of bioclastic texture with bioturbation. 6.A.Blade fragment of Urbasa Flint; B. Detail of bioclastic texture with a section of Nummulites heberti. 7.A. Blade of Treviño flint. B. Detail of bioclastic texture with ostracods. 8.A. Macroscopic aspect of a possible flint of Grand-Pressigny. 9.A. Macroscopic aspect of a possible flint of «Grain de Mil». 
y el yacimiento arqueológico. Teniendo en cuenta estas distancias, se han clasificado los sílex como:

- Local, aquel localizado en un radio de no más de $30 \mathrm{~km}$;

- Regional, aquel situado a entre 30 y 200 km de distancia; y

- Extra-regional, cuando la localización de un sílex sobrepasa los $200 \mathrm{~km}$.

Por lo tanto, partiendo de esta clasificación y haciendo un cálculo estimado aproximado de las distancias de los puntos de captación, se desprende que el sílex local es el de Flysch de Bidache, a casi $30 \mathrm{~km}$ de distancia, y que los sílex regionales serían el resto: Gaintxurizketa a escasos $40 \mathrm{~km}$, Salies de Béarn a unos 60 $\mathrm{km}$, Tercis a unos $70 \mathrm{~km}$, Chalosse a unos $90 \mathrm{~km}$, Urbasa a $110 \mathrm{~km}$ y Treviño a $180 \mathrm{~km}$, rozando este último los límites de la categoría extra-regional. Los sílex extra-regionales serían «Grain de mil», a aproximadamente más de 200 km de distancia, y Grand-Pressigny cuya localización superaría los 400 km.

\subsection{Modos de explotación de las variedades si- líceas, gestión diferenciada}

A la hora de clasificar los restos de la colección del nivel a estudiar se ha optado por diferenciar 6 grupos que encajarían con las fases de explotación de la materia prima (partiendo del concepto de fases de producción o de cadena operativa). En primer lugar y dentro de la fase de captación se situarían los núcleos, fragmentos brutos de sílex configurados para la extracción de soportes; en segundo lugar, los avivados que incluyen tanto los restos del proceso de pre-configuración como de configuración y retoque; en tercer lugar se encuentran los dos grupos de soportes, las lascas y las láminas; en cuarto lugar, los restos resultantes del proceso de talla y que en principio se desechan, incluyendo los fragmentos indeterminados o trozos (denominados aquí chunks); y por último, los restos que presentan retoque, los cuales se clasifican a su vez en grupos tipológicos.

Observando el gráfico (Figura 3) se percibe una distribución similar de los restos del proceso de talla para las cuatro principales fuentes: Bidache, Gaintxurizketa, Tercis y Chalosse. En todos ellos se da una mayor representación equilibrada entre los soportes brutos, lascas y láminas, y los débris o fragmentos menores. La información aportada por el sílex de Salies de Béarn no es representativa porque se trata de una variedad con apenas evidencias. Los sílex de Urbasa y de Treviño, a pesar de ser minoritarios, son un poco más numerosos que los de Salies de Béarn muestra un comportamiento típico de las materias primas aportadas desde distancias lejanas. Por un lado, Urbasa tiene un porcentaje relativo elevado de soportes retocados y de las fases de reacondicionamiento; y por otro lado, el sílex de Treviño presenta un predominio de soportes brutos junto con soportes retocados en menor medida.

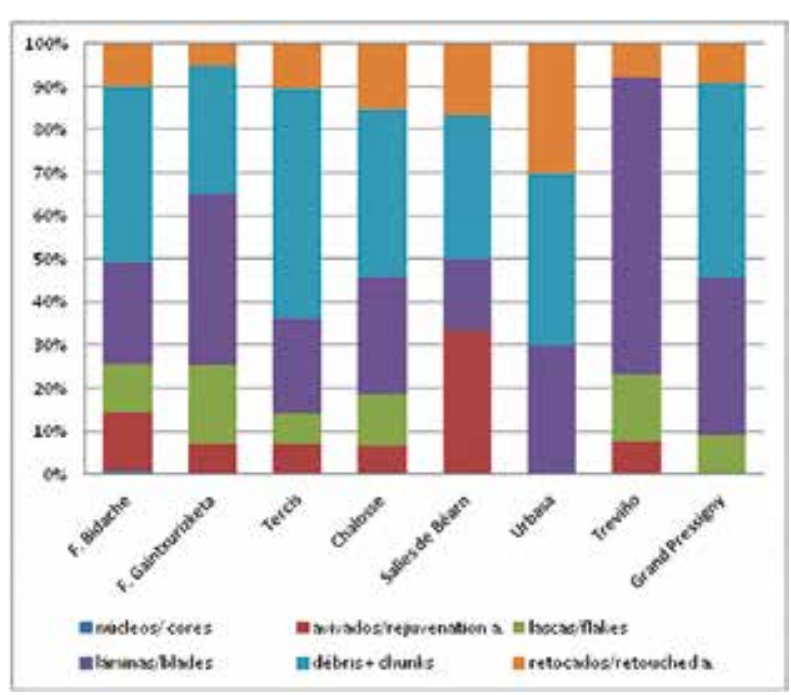

Fig. 3. Gráfico donde se observa la distribución de los elementos de la industria del nivel G de Berroberria (núcleos, avivados, lascas, láminas, débris y chunks y retocados) para las diferentes variedades silíceas identificadas / The graphic shows the distribution of the lithic assemblage (cores, rejuvenation, flakes, blades, débris and chunks and retouched artifacts) of level $\mathrm{G}$ of Berroberria interrelated with the identified flint types.

En cuanto al resto de tipos en general, añadir que se trata de una industria laminar que incluso puede comprobarse en la gestión de sílex lejanos como el de Treviño; las láminas aparecen en mayor porcentaje que las lascas en todos los casos.

La gestión de los sílex muestra diferencias bien si son sílex locales, regionales o extra-regionales. En el caso del tipo de Bidache, el mayoritario y de origen local, las fases de la cadena operativa están bien representadas desde las primeras fases de captación. Los únicos núcleos recogidos en el nivel G se confeccionaron sobre este sílex, al que habría que sumar uno en cristal de roca.

Las evidencias retocadas son significativas en relación con el debitado en los casos de los sílex más alejados a los que puede incluirse el único resto de «Grain de mil». Esta circunstancia se da en la mayoría de los yacimientos del Paleolítico Superior, por lo que Berroberria no sería un caso aparte. Como excepción, el sílex de Treviño y el posible Grand-Pressigny no seguirían este patrón; sin embargo, como se ha señalado con anterioridad los restos de debitado se relacionarían con una actividad concreta de reacondicionamiento sobre evidencias excepcionales que no han sido detectadas en el yacimiento.

En cuanto al uso del cristal de roca, ésta se ha explotado de forma intensiva y tiene representación a lo largo de las primeras fases de producción donde se incluye un núcleo; no obstante, son pocas las evidencias encontradas (un total de 17 restos de pequeño tamaño, 9 de ellos débris). Esta materia prima se consideraría de carácter local ya que presumiblemente se habría recogido en las inmediaciones del yacimiento y de forma casual. 
Si atendemos al conjunto de los retocados y a su clasificación podemos extraer mayor información acerca de las preferencias en empleos de determinadas materias primas para determinados artefactos, a través de la estadística. El método que emplearemos es la distribución $X^{2}$ (TARRIÑO, 2001a). En la tabla (Tabla I) aparecen los valores para $\chi^{2}$ : destacados en negrita aquellos que son heterogéneos, en cursiva y negrita aquellos heterogéneos con reservas, siendo el resto valores homogéneos. De esta forma la estadística muestra que la mayoría de los resultados son homogéneos. Entre los grupos heterogéneos destacan con superávit los abruptos, láminas de dorso truncadas y buriles sobre sílex de
Gaintxurizketa, mientras que las raederas lo hacen para las variedades de Chalosse y Urbasa. Por otro lado, el sílex de Tercis tiene una presencia significativa positiva en los elementos de dorso, puntas y láminas, lo que indicaría una elección no azarosa a la hora de la configuración de dichos artefactos sobre esta variedad silícea.

\subsection{Territorio de explotación}

Una vez reconocidos los puntos de captación de la materia prima de Berroberria se han proyectado sobre un mapa (Figura 4). Se observa el territorio de captación a nivel regional, alcanzando distancias de hasta

\begin{tabular}{|c|c|c|c|c|c|c|c|c|c|c|c|c|c|c|c|c|c|c|c|c|c|}
\hline & \multicolumn{3}{|c|}{ FLYSCH } & \multicolumn{3}{|c|}{ GAINTXURIZKETA } & \multicolumn{3}{|c|}{ CHALOSSE } & \multicolumn{3}{|c|}{ SALIES DE BÉARN } & \multicolumn{3}{|c|}{ URBASA } & \multicolumn{3}{|c|}{ TREVIÑO } & \multicolumn{3}{|c|}{ TERCIS } \\
\hline & $n$ & $\sigma$ & $x^{2}$ & $n$ & $\sigma$ & $x^{2}$ & $n$ & $\sigma$ & $x^{2}$ & $n$ & $\sigma$ & $x^{2}$ & $n$ & $\sigma$ & $x^{2}$ & $n$ & $\sigma$ & $x^{2}$ & $\mathrm{n}$ & $\Sigma$ & $x^{2}$ \\
\hline G & 2 & - & 1,36 & 0 & - & 5,415 & 0 & - & 0,137 & 0 & - & 11,43 & 0 & - & 1,347 & 0 & - & 11,43 & 0 & - & 0,187 \\
\hline $\mathrm{R}$ & 1 & - & 3,427 & 0 & - & 0,229 & 2 & + & 9,892 & 0 & - & 9,151 & 1 & + & 8,018 & 0 & - & 9,151 & 1 & + & 0,894 \\
\hline D & 5 & + & 3,475 & 0 & - & 0,229 & 0 & - & 0,351 & 0 & - & 9,151 & 0 & - & 8,433 & 0 & - & 9,151 & 0 & - & 0,478 \\
\hline LD & 59 & + & 0,978 & 1 & - & 6,868 & 7 & + & 0,576 & 1 & + & 0,514 & 1 & - & 1,479 & 1 & + & 0,514 & 8 & + & 10,912 \\
\hline LDT & 4 & + & 0,875 & 1 & + & 3,187 & 0 & - & 0,351 & 0 & - & 9,151 & 0 & - & 8,433 & 0 & - & 9,151 & 0 & - & 0,478 \\
\hline PD & 0 & - & 1,502 & 0 & - & 1,366 & 0 & - & 3,11 & 0 & - & 13,539 & 0 & - & 3,256 & 0 & - & 13,539 & 1 & + & 10,91 \\
\hline A & 0 & - & 4,57 & 1 & + & 6,417 & 0 & - & 0,207 & 0 & - & 3,256 & 0 & - & 3,018 & 0 & - & 3,256 & 0 & - & 0,282 \\
\hline $\mathrm{T}$ & 1 & - & 0,082 & 0 & - & 5,415 & 0 & - & 0,137 & 0 & - & 11,43 & 0 & - & 1,347 & 0 & - & 11,43 & 0 & - & 0,187 \\
\hline B & 13 & - & 0,78 & 3 & + & 4,532 & 0 & - & 2,053 & 0 & - & 0,215 & 1 & + & 0,523 & 0 & - & 0,215 & 2 & - & 0,008 \\
\hline Total $\mathbf{n}$ & \multicolumn{3}{|c|}{85} & & \multicolumn{3}{|c|}{9} & \multicolumn{3}{|c|}{ 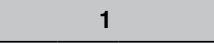 } & \multicolumn{3}{|c|}{3} & \multicolumn{3}{|c|}{1} & \multicolumn{3}{|c|}{12} \\
\hline$\%$ & \multicolumn{3}{|c|}{$72,6 \%$} & \multicolumn{3}{|c|}{$5,1 \%$} & \multicolumn{3}{|c|}{$7,7 \%$} & \multicolumn{3}{|c|}{$0,9 \%$} & \multicolumn{3}{|c|}{$2,6 \%$} & \multicolumn{3}{|c|}{$0,9 \%$} & \multicolumn{3}{|c|}{$10,2 \%$} \\
\hline
\end{tabular}

Tabla 1: Valores de $x^{2}$ para los grupos tipológicos (G: raspadores, R: raederas, D: denticulados, LD: láminas de dorso; LDT: láminas de dorso truncadas; PD: puntas de dorso; A: abruptos; T: truncaduras y B: buriles) del nivel G en relación con los tipos de sílex identificados. Se indica la desviación ( $\sigma$ ) de los valores, la cual es positiva (+) cuando existe superávit y negativa (-) cuando existe déficit. El número de evidencias se señala en la columna " $n$ ". Results of the statistic: $X^{2}$ values for the typological groups (G: end scrapers, R: side scrapers, D: denticulated, LD: backed blades, LDT: backed blades with, PD: backed points, A: abrupt, T: truncation and B: burins) of level G corresponding to the identified flint types. The deviation ( $\sigma)$ of the values is showed, which is positive (+) when exceeding and negative (-) when deficit. The quantity of evidences is marked out in column "n"

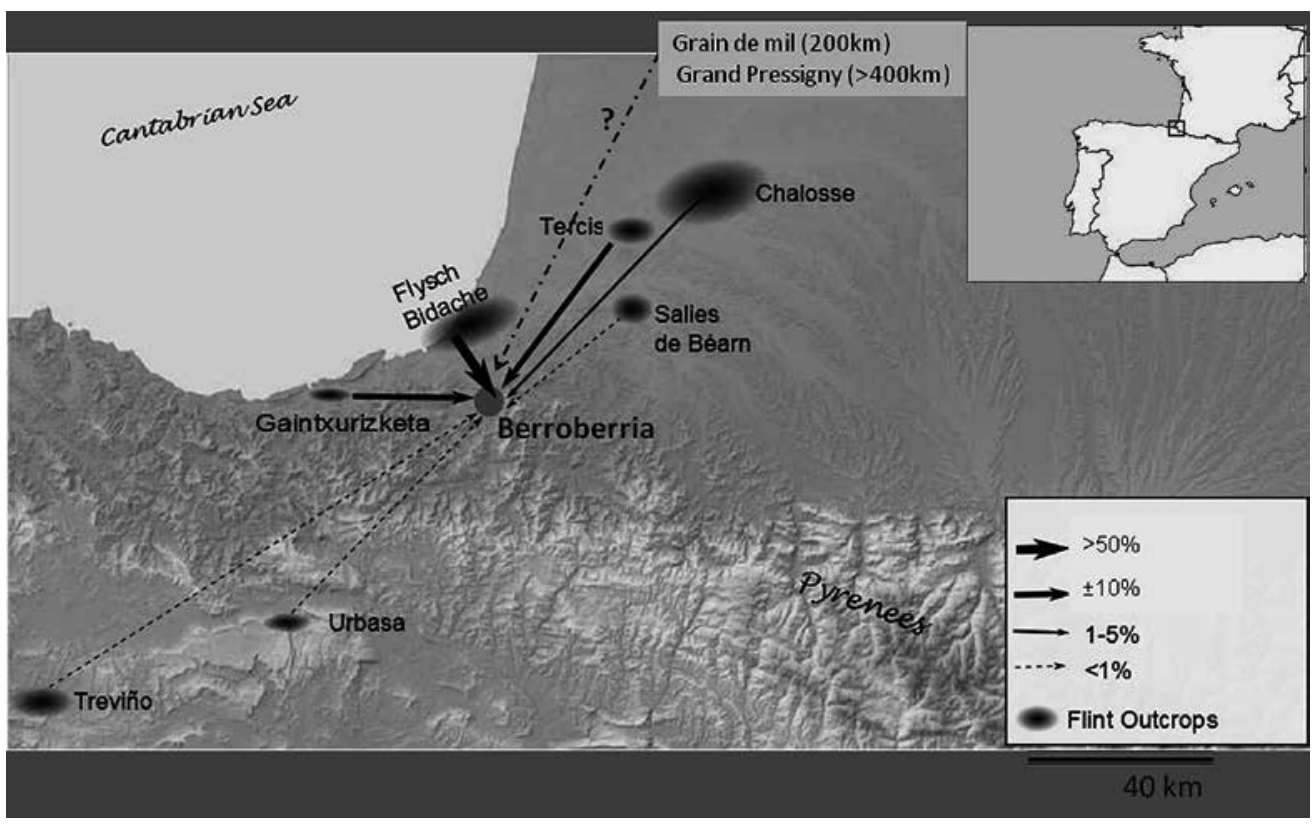

Fig. 4. Mapa del Pirineo Occidental con los principales afloramientos detectados en la industria lítica del yacimiento de Berroberria. Mediante flechas se indican los aportes en porcentajes de cada uno de los silex / Map of the Western Pyrenees pointing out the most relevant outcrops detected in the lithic industry of Berroberria site. The arrows indicate the percentages of use of each of the flints. 
$160 \mathrm{~km}$; por lo tanto, estaríamos hablando de amplias zonas de control. En el mapa se aprecia cómo es la aportación de las distintas variedades al yacimiento, pudiéndose comprobar que los sílex del sur tienen unas proporciones muy pequeñas y que el afloramiento más cercano es el que más aportes realiza. Las materias primas de origen noraquitano se han señalado mediante una línea discontinua y con interrogación, dado que se deberían hacer más analíticas para afirmar con fiabilidad esta procedencia y entre los restos indeterminados podrían aparecer más evidencias.

La procedencia lejana de variedades silíceas demuestra una amplia movilidad. Rasgo que como se ha indicado es característico del Paleolítico Superior (GENESTE, 1991). Sin embargo no puede afirmarse de qué tipo de movimientos se trata, bien sean por desplazamiento de los grupos humanos en busca de recursos o bien por llegada de otros grupos humanos de larga distancia. Por otro lado, sí puede asegurarse la búsqueda de materias primas de calidad en la configuración de la industria lítica. Los tipos silíceos son los mismos que han sido empleados en varios de los yacimientos de la zona y presentan unas propiedades buenas para la talla.

\section{CONCLUSIONES}

Gracias al estudio arqueopetrológico de su nivel G se corrobora que Berroberria se trata de un yacimiento con una gran variedad en cuanto a los aportes de diferentes tipos de sílex. Se han identificado un total de 7 tipos de sílex con seguridad: Flysch de Bidache, Flysch de Gaintxurizketa, Chalosse, Tercis, Salies de Béarn, Urbasa y Treviño, localizados tanto al norte (Cuenca de Aquitania) como al sur (Cuenca Vasco-Cantábrica) de los Pirineos. A los que hay que añadir los posibles tipos de «Grain de mil» y Grand-Pressigny procedentes del norte. Ello hace que el territorio de explotación del yacimiento durante el Magdaleniense Medio fuera más allá del territorio más inmediato, demostrando una movilidad amplia de los grupos que lo habitaban. El empleo de estas materias primas silíceas es similar al identificado en yacimientos cercanos de las mismas fechas.

También a modo de recapitulación destacar la presencia de sílex lejanos o extra-regionales a más de 200 km en dirección norte, como presencia inédita en yacimientos tan al sur de Aquitania. No obstante, son datos que habría que contrastar con otros yacimientos circundantes y sería preciso realizar más análisis para caracterizar estas rocas silíceas como ya se ha mencionado.

El yacimiento de Berroberria colabora así en el conocimiento sobre la gestión de las materias primas líticas, mostrando una explotación de los recursos locales con la inclusión de otros de origen más lejano. Este comportamiento entra dentro de la dinámica del Pirineo Occidental donde los sílex trazadores son los más explotados. En este caso el sílex local de Bidache tiene una explotación intensiva que se observa en toda la representación de las fases de la cadena operativa.

\section{AGRADECIMIENTOS}

Este trabajo ha sido llevado a cabo con el apoyo del proyecto PalMesoPyr y "Grupo Consolidado de Investigación en Prehistoria (IT-622-13) Área de Prehistoria, Dpto de Geografía, Prehistoria y Arqueología (Universidad del País Vasco, UPV/EHU). Agradecer también a los profesores A. Cava e I. Barandiarán por permitir el acceso y estudio al material arqueológico.

\section{BIBLIOGRAFÍA}

ARRIZABALAGA, A., CALVO, A., ELORRIETA, I., TAPIA, J., TARRIÑO, A

2014 Where to and what for? Mobility patterns and the management of lithic resources by Gravettian hunter-gatherers in the Western Pyrenees. Journal of Anthropological Research 70, 233-261.

\section{BARANDIARÁN, I.}

1979 Excavaciones en el covacho de Berroberría (Urdax): campaña de 1977. Trabajos de arqueología Navarra 1, 11-60.

1990 Revisión estratigráfica de Berroberria: Datos en 1990. Veleia 7, 7-34.

1993 Cueva de Berroberría (Urdax): informe de las campañas de excavación V (1990), VI (1991), VII (1992) y VIII (1993). Trabajos de arqueología Navarra 11, 243-247.

BARANDIARÁN, I., CAVA, A., AGUIRRE, M.

2013 El taller de sílex de Mugarduia Sur: una ocupación de Urbasa (Navarra) durante el Gravetiense. Servicio Editorial de la Universidad del País Vasco.

BON, F., CHAUVAUD, D., DARTIGUEPEYROU, S., GARDÈRE, $\mathrm{PH}, \mathrm{KLARIC}, \mathrm{L}$., MENSAN, R.

2002 Les ressources en silex de la Chalosse centrale: gîtes et ateliers du dôme diapir de Bastennes-Gaujacq et de l'anticlinal d'Audignon, en: CAZALS, N. (Ed.) Comportements techniques et économiques des sociétés du Paléolithique supérieur dans le contexte pyrénéen. Projet Collectif de Recherche Service Régional de l'Archéologie de Midi-Pyrénées, 47-63.

BORDES J-G

2002 Les interstratifications Châtelperronien / Aurignacien du Roc-de-Combe et du Piage (Lot, France). Analyse taphonomique des industries lithiques; implications archéologiques. Thèse de doctorat. Bordeaux: Université Bordeaux 1.

CAVA, A., BARANDIARÁN, I. (Eds.)

2008 Cazadores y tallistas en el abrigo de Portugain, una ocupación de Urbasa durante el Tardiglaciar. Fundación José Miguel de Barandiaran.

CHALARD, P., DUCASSE, S., BON, F., BRUXELLES, L., TEYSSANDIER, N., RENARD, C., GARDERE, P., GUILLERMIN, P., LACOMBE, S., LANGLAIS, M., MENSAN, R., NORMAND, C., SIMONNET, R., TARRIÑO, A.

2010 Chalosse-type Flint: exploitation and distribution of a Lithologic tracer during the Upper Paleolithic, Southern France, en: BREWER- LAPORTA, M., BURKE, A., FIELD, D. (Eds.) Ancient Mines and Quarries: a Trans-Atlantic Perspective. 13-22. Oxbow books, Oxford. 
DUNHAM, R.-J.

1962 Classification of carbonate rocks according to the depositional texture, en: HAM W. (Ed.) Memorie of the American Association of petroleum geologists, Classification of carbonate rocks. 108-121. Tulsa.

\section{ELORRIETA, I.}

2014 Los artefactos retocados del nivel C4d1j de Isturitz: estudio arqueopetrológico desde la Tipología Analítica, en: Seis décadas de Tipología Analítica. Actas en homenaje a Georges Laplace. 156-166. Vitoria-Gasteiz.

FEBLOT-AUGUSTINS, J.

1997 La circulation des matières premières au Paléolithique. ERAUL, $n^{\circ} 74$, Lieja.

\section{FERNÁNDEZ-ERASO, J., GARCÍA-ROJAS, M.}

2013 Tipología Analítica, en: GARCíA-DíEZ, M., ZAPATA, L. (Eds.) Métodos y técnicas de análisis y estudio en Arqueología Prehistórica. De lo técnico a la reconstrucción de los grupos humanos. 470-497. Universidad del País Vasco.

JAUBERT J., HUBLIN J-J., MC PHERRON S., SORESSI M., BORDES J-G., CLAUD E., COCHARD D., DELAGNES A., MALLYE J-B., MICHEL A., NICLOT M., NIVEN L., PARK S-J., RENDU W., RICHARD M., RICHTER D., ROUSSEL M., STEELE T., TEXIER J-P. et THIEBAUKT C.

2008 Paléolithique moyen et Paléolithique supérieur ancien à Jonzac (Charente-Maritime): premiers résultats des campagnes 2004-2006, en: JAUBERT J., BORDES J-G., ORTEGA I. (Ed.) Les sociétés du Paléolithique dans un grand Sud-Ouest: nouveaux gisements, nouveaux résultats, nouvelles méthodes. Mémoires de la Société Préhistorique Française, t. XLVII, 203-343.

GENESTE, J-M.

1991 L'approvisionnement en matières premières dans les systèmes de production lithique: la dimension spatiale de la technologie. Tecnología y cadenas operativas líticas: Bellaterra, del 15 al 18 de enero de 1991. Treballs d'Arqueologia 1, 1-36.

LAPLACE, G.

1973 La typologie analytique et structurale : Basse rationnelle d'étude des industries lithiques et osseuses. Banques de Données Archéologiques 932, 91-143.

\section{MILLET, D., MALLET, N., GIOT, D.}

1986 Les silex de la région du Grand-Pressigny (Indre-et-Loire). Recherche géologique et analyse pétrographique. Revue archéologique du Centre de la France, vol. 25, $\mathrm{n}^{\circ} 25-1,21-36$.

NORMAND, C.

2002 Les ressources en matières premières siliceuses dans la basse vallée de l'Adour et de ses affluents, en: CAZALS, N. (Ed.) Comportements techniques et économiques des sociétés du Paléolithique supérieur dans le contexte pyrénéen. Project Collectif de Recherche 2002. Service Régional de l'Archéologie de Midi-Pyrénées, 26-46.
PRIMAULT, J.

2003 Exploitation et diffusion des silex de la région du Grand-Pressigny au Paléolithique. Thése Université de Paris X-Nanterre.

\section{SIMONNET, R.}

1982 Grandes lames de silex dans le Paléolithique supérieur des Pyrénées centrales. Essai sur des documents marginaux. Préhistoire Ariègeoise - Bulletin de la Société Préhistorique de l'Ariège 37, 61-106.

TARRIÑO, A

2001a El sílex en la Cuenca Vasco-Cantábrica y el Pirineo Navarro: caracterización y su aprovechamiento en la Prehistoria. Tesis Doctoral. Universidad del País Vasco.

2001b Procedencia de los sílex de la industria lítica del yacimiento de Aizpea (Arive, Navarra), en: BARADIARÁN, I., CAVA, A. (Eds.) Cazadores-recolectores en el Pirineo navarro, El sitio de Aizpea entre 8000 y 6000 años antes de ahora. Veleia, Anejos, Series Maior, vol. 10 51-61.

TARRIÑO, A., ELORRIETA, I.

2012 La explotación de los recursos abióticos durante el Gravetiense cantábrico. Primeros datos sobre el Pirineo occidental y la Cuenca Vasco-Cantábrica, en: DE LAS HERAS, C., LASHERAS, J.A., ARRIZABALAGA, A., DE LA RASILLA, M. (Eds.) Pensando el Gravetiense: nuevos datos para la región cantábrica en su contexto peninsular y pirenaico, Monografía 23. 330-346. Museo Nacional y Centro de Investigación de Altamira.

\section{TARRIÑO, A., ELORRIETA I, GARCÍA-ROJAS, M.}

2015 Flint as raw material in prehistoric times: Cantabrian Mountain and Western Pyrenees data. Quaternary International 364, 94-108.

TARRIÑO, A., NORMAND, C.

2002 Procedencia de los restos líticos en el Auriñaciense Antiguo (C4b1) de Isturitz (Pyrénées-Atlantiques, Francia). Espacio, Tiempo y Forma, Serie I, Prehistoria y Arqueología 15, 145-153 